\title{
THE RUIN PROBABILITY IN A SPECIAL CASE
}

\section{H. Bohman}

Stockholm

It is fantastic how the computer has changed our attitude to numerical problems. In the old days when our numerical tools were paper, pencil, desk calculator and logarithm tables we had to stay away from formulas and methods which led to too lengthy calculations. A consequence is that we have a tendency to think of numerical analysis in terms of the classical tools. If we go back to the results of earlier writers it seems, however, very likely that many results and formulas developed by them which had earlier a theoretical interest only could nowadays be applied successfully in numerical analysis.

As an example I take the ruin probability $\psi(x)$. The Laplace transform of $\psi(x)$ is given by the following expression

$$
\int_{0}^{\infty} \psi(x) e^{-y x} d x=\frac{\mathrm{I}}{y}+\frac{c-\mathrm{I}}{\mathrm{I}-c y-p(y)}
$$

where $c>\mathrm{I}$. In fact $(c-\mathrm{I})$ is equal to the "security loading". The function $p(y)$ is equal to the Laplace transform of the claim distribution. We assume that the mean claim amount is equal to one, i.e. $p^{\prime}(0)=-\mathrm{I}$.

In his book from I955 [I] Cramér points out that this formula will be more easy to handle if the claim distribution is an exponential polynomial. In this case we have

$$
p(y)=\sum_{1}^{N} \frac{a_{n}}{\mathrm{I}+b_{n} y}
$$

where

$$
\begin{gathered}
\Sigma a_{n}=\mathrm{I} \\
\Sigma a_{n} b_{n}=\mathrm{I} \\
a_{n}>0 \\
b_{n}>0
\end{gathered}
$$


Cramér's results are given on pages $8 \mathrm{I}-83$ in his book. We reproduce them here with a slight change of notations only.

First we write $p(y)$ in the following form

$$
p(y)=\mathrm{I}-y \sum_{1}^{N} \frac{q_{n}}{\mathrm{I}+b_{n} y}
$$

where $q_{n}=a_{n} b_{n}$.

For the Laplace transform we then get the following expression

$$
\frac{\mathrm{I}}{y}-\frac{c-\mathrm{I}}{c y-y \sum \frac{q_{n}}{\mathrm{I}+b_{n} y}}
$$

It follows from Cauchy's theorem that this is equal to

$$
\sum \frac{C_{n}}{y-r_{n}}
$$

where $r_{1}, r_{2}, \ldots, r_{N}$ are the roots of the equation

$$
\begin{gathered}
c=\sum_{1}^{N} \frac{q_{n}}{\mathrm{I}+\frac{b_{n} y}{}} \\
C_{m}=\frac{c-\mathrm{I}}{\sum_{n} \frac{q_{n}}{\left(\mathrm{I}+b_{n} \gamma_{m}\right)^{2}}-c}
\end{gathered}
$$

From this expression for the Laplace transform of $\psi(x)$ follows that $\psi(x)$ itself is equal to

$$
\psi(x)=\sum_{1}^{N} C_{n} e^{r_{m} x}
$$

The essential difficulty here is to find the roots $r_{m}$. This is very easy to do with the aid of the computer. You must probably have a rootextracting subroutine as part of your subroutine package. If not, the following procedure is recommended in the present case. 
Between $-\mathrm{I} / b_{n}$ and $-\mathrm{I} / b_{n+1}$ there is one real root. Assuming that $b_{n+1}>b_{n}$ we put

$$
\begin{aligned}
x_{1} & =-\mathrm{I} / b_{n} \\
x_{2} & =-\mathrm{I} / b_{n+1} \\
\longrightarrow x & =\frac{x_{1}+x_{2}}{2} \\
F & =c-\sum \frac{q_{n}}{\mathrm{I}+b_{n} x} \\
& \text { If } F>\text { o put } x_{2}=x, \text { else put } x_{1}=x
\end{aligned}
$$

Repeat this procedure until $\left(x_{2}-x_{1}\right)$ is small enough. Then you have $r_{n}=x$ with the prescribed precision.

As an example I choose

$$
\begin{aligned}
& N=5 \\
& b_{n}=\mathrm{IO}^{n-1} \cdot 0.22222 \\
& a_{n}=10^{-n} / 0 . \mathrm{IIIII} \\
& q_{n}=0.2
\end{aligned}
$$

Then

$$
\begin{array}{ll}
r_{1}=-3.70384 & C_{1}=0.01889 \\
r_{2}=-0.35 \mathrm{I} 67 & C_{2}=0.03004 \\
r_{3}=-0.03247 & C_{3}=0.05206 \\
r_{4}=-0.00278 & C_{4}=0.10966 \\
r_{5}=-0.00015 & C_{5}=0.63 \mathrm{I} 67
\end{array}
$$

It follows that

$$
\begin{aligned}
& \psi(\text { IO } 000)=0 . \text { I } 4 \mathrm{I} \\
& \psi(20000)=0.03 \mathrm{I} \\
& \psi(30000)=0.007 \\
& \psi(40000)=0.002
\end{aligned}
$$

\section{REFERENCE}

[I] Cramér, Harald: Collective Risk Theory, I955 (The I 955 jubilee volume of Skandia Insurance Company). 